\title{
A Novel Approach to Shape Decomposition and Representation using Soft Morphological Filters
}

\author{
N.Santhi \\ (Research Scholar, Dr.M.G.R. Educational and \\ Research Institute, Maduravoyal, Chennai) \\ Associate Professor, Dept. of ECE, \\ Noorul Islam Centre for Higher Education, \\ Tamil Nadu, India
}

\author{
Dr.K.Ramar \\ Principal \\ Srividya College of Engineering and Technology, \\ Virudhunagar, Tamil Nadu, India.
}

\begin{abstract}
Shape decomposition and reconstruction are vital factors in image processing and analysis applications. A generalized skeleton transform allows a shape to be represented as a collection of modestly overlapped octagonal shape parts. One of the main problems with the existing algorithms is that they generate noise after decomposition. For ordinary images the rate of noise may not be effective but it will be more when applied on printed or handwritten characters. In this paper we have introduced a novel algorithm to tackle this issue by applying a soft morphological filter (SMF) after morphological decomposition. The algorithm was applied on various types of decomposition images. The experimental results indicated that the present decomposition algorithm produces images with more clarity when compared with other algorithms.
\end{abstract}

\section{Keywords}

Mathematical Morphology, Structuring elements, Shape decomposition, Soft Morphological filters (SMF).

\section{INTRODUCTION}

SHAPE representation or analysis [1] is an important issue in the many applications of machine vision, such as Character recognition, contour matching for medical imaging reconstruction \& industrial inspection .Many other visual tasks also can be achieved by shape recognition. Shape representation provides the foundation for shape analysis applications such as shape matching, object recognition, image coding etc. In the recent years a number of morphological shape representation algorithms have been proposed. A class of recursive transformations were introduced [2].The main distinction of the recursive transformation is that the pixel's value of the transformed image depend upon the pixel's values of both the input image and the transformed image.

Morphological shape decomposition $[3,4,5]$ is yet another approach, which is a popular method for shape representation but this approach lacks robustness especially in impulsive noise. Shape representation scheme using morphological operations [6,7] is another method in which a given shape is decomposed into a union of certain disks contained in the shape. The overlapping between the representative disks is completely eliminated. This method is only suitable for serial implementations. Morphological shape decomposition algorithm $[8,9]$ decomposes a two-dimensional (2-D) binary shape into a collection of convex polygonal components. A single convex polygonal approximation for a given image is first identified. This first component is determined incrementally by selecting a sequence of basic shape primitives. These shape primitives are chosen based on shape information extracted from the given shape at different scale levels. Another new class of morphological operations [10,11], allows one to select varying shapes of structuring elements. However, the sweep erosion and dilation do not satisfy the basic properties of mathematical morphology. Morphological shape decomposition algorithm uses a set of representative disks [12].These representative disks are sensitive to small boundary changes as skeleton based representations. Skeletonization of $2 \mathrm{D}$ images is based on primitive concepts of morphology. This approach is also sensitive to boundary noise .Recursive order -static soft morphological filters [13] provide excellent performance in noise reduction. Soft Morphological filter [14] is simple and efficient.

\section{SOFT MORPHOLOGICAL SHAPE REPRESENTATION}

In this paper we are introducing a new algorithm for efficient shape representation using soft morphological filters. A good shape representation scheme provides a good foundation for shape analysis . Skeletonization is the first step to represent any shape .By using this the skeleton of a given shape will be found. Then using these skeleton points shape reconstruction will be done . In the reconstruction some noise will exist. To get the noise free output image we introduce soft morphological filters for filtering process .While applying the soft morphological filters in the reconstructed shape image we will get highly efficient shape representation. In our new algorithm we developed efficient shape representation by using the following three steps.

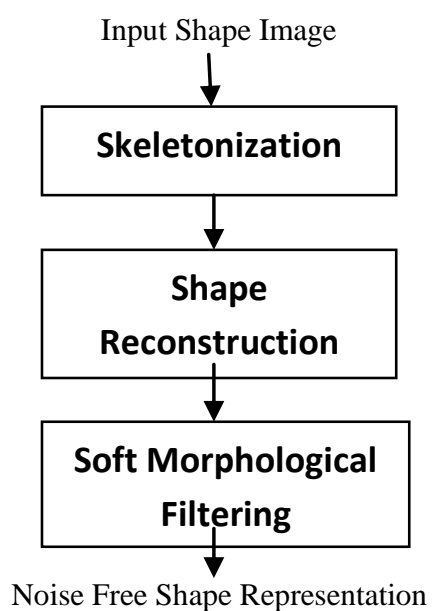

Fig 1.Block diagram 


\subsection{Skeletonization}

Skeletonization is a morphological operation that is used to remove selected foreground pixels from shape images. Shape analysis can be done easily by this skeletonization process. To reduce the amount of data and time to be processed this skeletonization is needed .In our algorithm we use eight structuring elements to derive the skeleton points which is shown in the fig.1. The eight structuring elements will be applied in cyclic sequence.

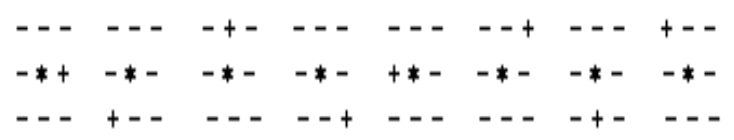

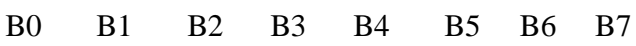

Fig.1 Eight two-point structuring elements

By repeatedly applying soft erosion operation using the above mentioned eight two-point structuring elements we get the skeleton output which is shown in the fig. 2

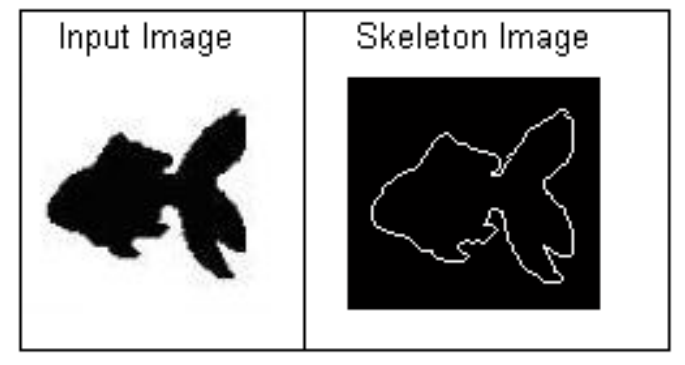

Fig.2 Skeletonization

\subsection{Shape Reconstruction}

Reconstructing a shape into meaningful representation plays a strong role in shape related applications. After skeletonization by using the skeleton points, reconstruction will be done. In our paper a new algorithm was developed which is shown in below.

\section{Algorithm :}

1. Read number of received skeleton points in $\mathrm{N}$ and initialize I=1 and initialize an array,'IM ' with zeros

2. Read the first skeletal point coordinate and the corresponding structuring element

3. Place 1 at the coordinate position and dilate with the corresponding structuring element.

4. If $\mathrm{I}<=\mathrm{N}$ read the next skeletal coordinate and the corresponding structuring element

5. Initialize array (X) with zeros and place 1 at the coordinate position and dilate with the corresponding structuring element.

6. Then add (X) to (IM) and I=I+1, then go to step 4 .

7. If the condition $\mathrm{I}<=\mathrm{N}$ is false, print (IM) and get the reconstructed shape image.
The reconstructed shape image output is shown in fig. 3

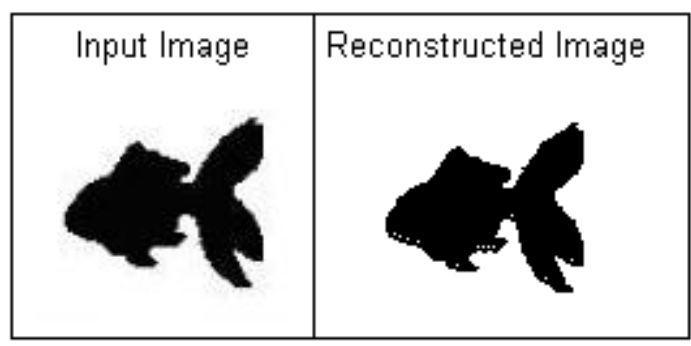

Fig.3 Reconstructed Shape Image

\subsection{Soft Morphological Filtering}

Soft morphological filtering is the extension of standard morphological filtering. To reduce noise effectively soft morphological filters are used. The main advantage of using soft morphological filtering method is it provide better smoothing capabilities and take less computational time. The soft morphological filters have two main properties. They are,1.less sensitive to additive noises 2 . Small variations in the shape to be filtered

In our algorithm in the reconstructed shape image to eliminate the noise Soft morphological filters are used to prevent any change in the image content .In image processing every pixel in the image is very important. There are more filters but during filtering process they affect the image content also. The below output fig. 4 shows the soft morphological filtered image.

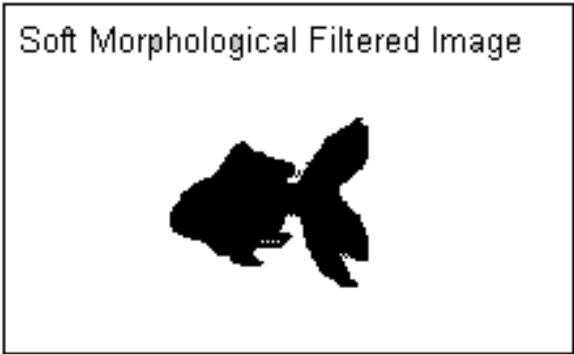

Fig.4 Soft Morphological Filtered Image

The advantages of soft morphological filters are ,High efficiency, Low complexity algorithm and restoration of noisy images is effective. The median filtered image and standard morphological filtered image are also shown in fig.5.They reduce noise as well as some shape content.

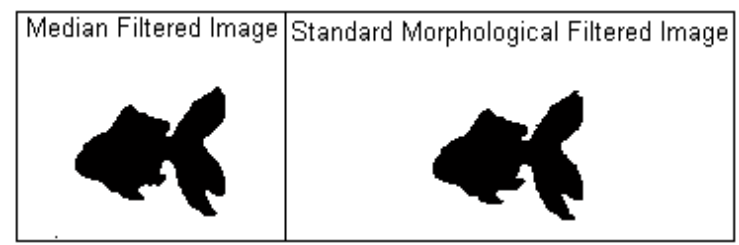

Fig.5 Filtered images 


\section{EXPERIMENTS}

Our soft morphological shape representation algorithm was tested on nine shape images shown in Fig.6.Fig 7 shows the reconstructed images. To compare our algorithm with the existing algorithms, we calculated the number of error functions. Applying the equations shown below, the error functions were calculated.

Let:

$f(x, y)=$ input shape image

$\mathrm{g}(\mathrm{x}, \mathrm{y})=$ reconstructed image

$\mathrm{M}$ and $\mathrm{N}=$ The sizes of input and reconstructed image

\section{Error functions:}

1. AEPP: Average error per pixel

$$
\mathrm{AEPP}=\frac{1}{\mathrm{M} \times \mathrm{N}} \sum_{\mathrm{i}=0}^{\mathrm{M}-1} \sum_{\mathrm{j}=0}^{\mathrm{N}-1} \mid \mathrm{f}(\mathrm{x}, \mathrm{y})-\mathrm{g}(\mathrm{x}, \mathrm{y})
$$

2. MSE: Mean square error

$$
\operatorname{MSE}=\frac{1}{M \times N} \sum_{i=0}^{M-1} \sum_{j=0}^{N-1}(f(x, y)-g(x, y))^{2}
$$

3. RMSE: Root mean square error

$$
\text { RMSE }=\sqrt{\frac{1}{M \times N} \sum_{i=0}^{M-1} \sum_{j=0}^{N-1}(f(x, y)-g(x, y))^{2}}
$$

4. SNR (ms) : Signal to noise ratio (mean square)

$$
\operatorname{SNR}(\mathrm{ms})=\frac{\sum_{\mathrm{i}=0}^{\mathrm{M}-1} \sum_{\mathrm{j}=0}^{\mathrm{N}-1} \mathrm{~g}(\mathrm{x}, \mathrm{y})^{2}}{\sum_{\mathrm{i}=0}^{\mathrm{M}-1} \sum_{\mathrm{j}=0}^{\mathrm{N}-1}(\mathrm{~g}(\mathrm{x}, \mathrm{y})-\mathrm{f}(\mathrm{x}, \mathrm{y}))^{2}}
$$

5. SNR (rms): Signal to noise ratio (root mean square)

$$
\operatorname{SNR}(\mathrm{rms})=\sqrt{\frac{\sum_{\mathrm{i}=0}^{\mathrm{M}-1} \sum_{\mathrm{j}=0}^{\mathrm{N}-1} \mathrm{~g}(\mathrm{x}, \mathrm{y})^{2}}{\sum_{\mathrm{i}=0}^{\mathrm{M}-1} \sum_{\mathrm{j}=0}^{\mathrm{N}-1}(\mathrm{~g}(\mathrm{x}, \mathrm{y})-\mathrm{f}(\mathrm{x}, \mathrm{y}))^{2}}}
$$

6. PSNR: Peak signal to noise ratio

$$
\text { PSNR }=10 * \log _{10}\left(\frac{255^{2}}{\mathrm{MSE}}\right)
$$

7. Error-Rate: Error-rate per pixel

$$
\text { Error_Rate }=\frac{\sum_{\mathrm{i}=0}^{\mathrm{M}-1} \sum_{\mathrm{j}=0}^{\mathrm{N}-1} \text { If }(\mathrm{x}, \mathrm{y})-\mathrm{g}(\mathrm{x}, \mathrm{y}) \text { | }}{\sum_{\mathrm{i}=0}^{\mathrm{M}-1} \sum_{\mathrm{j}=0}^{\mathrm{N}-1} \mathrm{f}(\mathrm{x}, \mathrm{y})}
$$

The error calculations are shown in Table. 1 and Table. 2

Table.1

\begin{tabular}{|c|l|l|l|l|}
\hline & AEPP & MSE & RMSE & SNR(ms) \\
\hline Digits & 177.061 & 221.09 & 14.86 & 1.0150 \\
\hline Butterfly & 190.540 & 214.81 & 14.65 & 1.0081 \\
\hline Dog & 191.548 & 218.39 & 14.77 & 1.0084 \\
\hline Fish & 168.837 & 197.94 & 14.06 & 1.0072 \\
\hline Lamb & 179.092 & 192.52 & 13.87 & 1.0037 \\
\hline Letters & 187.487 & 211.37 & 14.53 & 1.0129 \\
\hline elephone & 178.382 & 195.38 & 13.97 & 1.0068 \\
\hline Tree & 165.429 & 255 & 15.96 & 1 \\
\hline
\end{tabular}

Table.2

\begin{tabular}{|c|c|c|c|}
\hline & SNR(rms) & PSNR & Error Rate \\
\hline Digits & 1.0075 & 24.6843 & 1.0724 \\
\hline Butterfly & 1.0041 & 24.8117 & 1.1231 \\
\hline Dog & 1.0042 & 24.7383 & 1.1672 \\
\hline Fish & 1.0036 & 25.1660 & 1.0313 \\
\hline Lamb & 1.0019 & 25.2863 & 1.9680 \\
\hline Letters & 1.0064 & 24.8806 & 1.8383 \\
\hline Telephone & 1.0034 & 25.2231 & 1.9436 \\
\hline Tree & 1 & 24.0654 & 3.3246 \\
\hline
\end{tabular}

The key difference between our algorithm and the error free decomposition algorithm is we use soft morphological filter for the removal of noise for the reconstruction of images. Otherwise, the two algorithms are very similar. However, this addition of SMF provided

much power. And the power is obtained with very low cost. In our shape representation algorithm, first the skeleton points were generated.

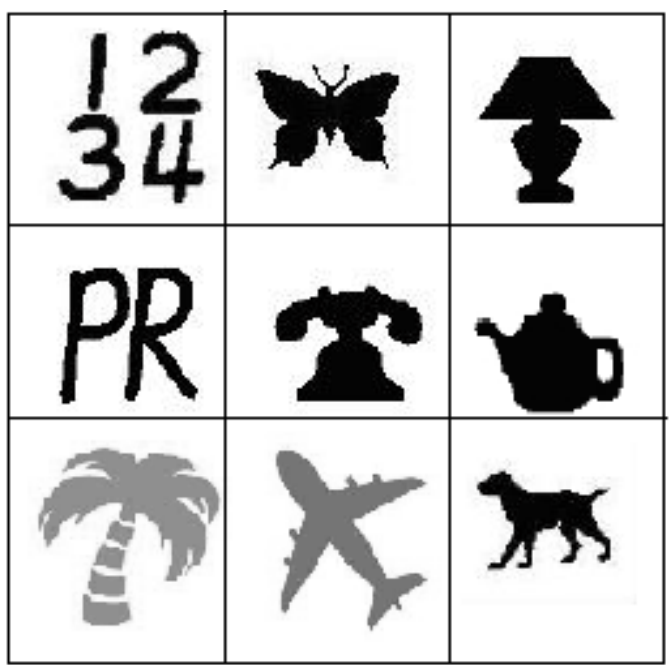

Fig.6 Input Shape Images

Then noise was removed by applying soft morphological filter. From this point of view, the computation procedure of our algorithm is significantly no more complex than that of the existing algorithms. Shape Images used in our algorithm is shown in fig.6.In our paper ten shape images are tested.fig.7 shows the reconstructed shape images. The soft morphological filtered image is shown in fig. 8 . 


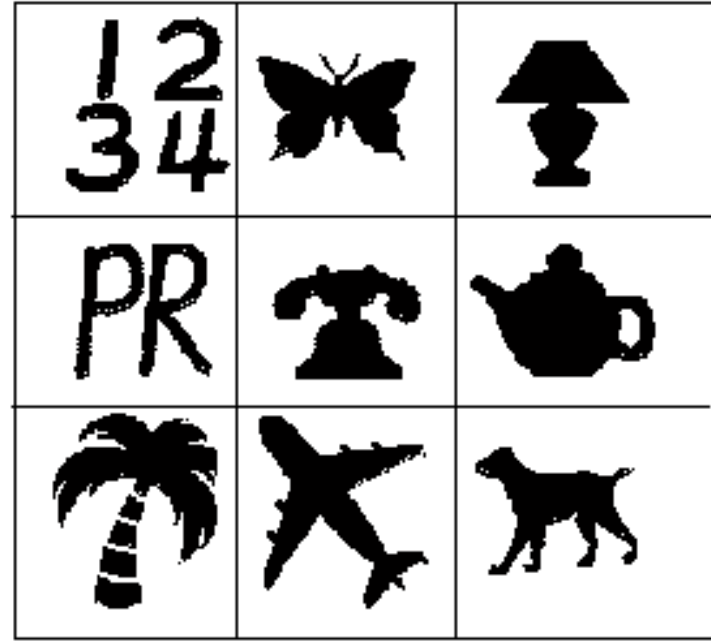

Fig.7 Reconstructed Shape images

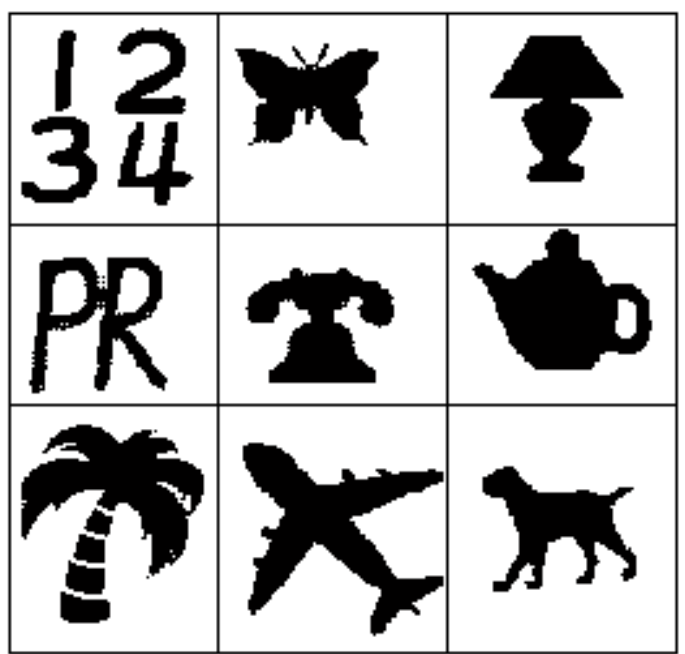

Fig.8 Soft morphological filtered image

\section{CONCLUSION}

In this paper, we have developed a new morphological shape representation algorithm using soft morphological filters. By this noise rate is reduced. The proposed algorithm is more efficient than the existing shape representation algorithms. The soft morphological filtering produces good solution to the noise reduction. The algorithm is very simple and easy to implement. The more efficient and the more precise representations generated by the new algorithm can often be considered more natural as well. It could be used for shape analysis and processing applications.

\section{ACKNOWLEDGEMENT}

The author wishes to thank the referees for their very helpful comments.

\section{REFERENCES}

[1] J.Xu,2001 " Morphological representation of 2-D binary shapes using rectangular components", Pattern Recog.,34:277-286.DOI:10.1109/ICP-1999.823020.

[2]. J. Serra, "Image Analysis and Mathematical Morphology". London, U.K.: Academic, 1982.
[3] I. Pitas and A. N. Venetsanopoulos, "Morphological shape decomposition,"IEEE Trans. Pattern Anal. Mach. Intell., vol. 12, no. 1, pp. 38-45, Jan. 1990

[4] Vijayakumar et al., 2008.An improved iterative morphological decomposition approach for image skeletonzation .GVIP.J.,8:47-54.

[5] Xu,.J.,2007.Morphological decomposition of 2-D binary shapes into modestly overlapped octagonal and disk components.IEEE trans.image Process.,16:337348.DOI:10.1109/TIP.2006.888328

[6] Demin Wang ${ }^{\ddagger}$, Veronique Haese-Coat ${ }^{\ddagger}$ and Joseph Ronsin "Shape decomposition and representation using a recursive morp341 hological operation" Laboratoires d'Automatique, Équips Image, Institute National des Sciences Appliqués, France March 1995

[7] V. Vijaya Kumar, et. al., "A new skeletonization method based on connected component approach," International Journal of Computer Science and Network Security, vol.8 no.2, pp.133-137, 2008.

[8] J.Xu,"Efficient Morphological shape representation with overlapping disk components," IEEE Transactions on image processing,vol 10,no.9,2001.

[9] Vijayakumar kumar,V. et al .,2006. A comparison on morphological skeleton transform with multiple structuring elements.pg.no-8.Proceedings of the international Conference on ICORG,june 6-8,NIRD campus,Hyderabad.

[10] Frank Y. Shih, Vijayalakshmi Gaddipati “Geometric modeling and representation based on sweep mathematical morphology" Information Sciences-Informatics and Computer Science: An International Journal , Volume 171 Issue 1-3 March 2005.

[11] J.Xu,2003.A generalized discrete morphological skeleton transform with multiple structuring elements for the extraction of structural shape components.IEEE trans.image process.,12:16771686.DOI:10.1109/TIP.2003.819225.

[12] V.Vijayakumar, et al.,"Error free iterative morphological decomposition Algorithm for shape Represention",international Journal of Computer Science and network Security,pp.71-78,2009.

[13] S.C. Pei, C.L. Lai, F.Y. Shih "Recursive order-statistic soft morphological filters"IEE Proc.-Vis. Image Signal Process., Vol. 145, No. 5, October 1998

[14] Xu,J.,1998.Efficient morphological shape representation without searching. Proceedings of the IEEE International Conference on Image processing. Oct.4-7, IEEE Computer Society, Washington. DC.,USA., pp:262266.DOI:10.1109/ICIP.1998.723360 